\title{
ON THE IRREDUCIBLITY OF THE REPRESENTATION OF THE PURE BRAID GROUP ON THREE STRANDS
}

\author{
Hasan A. Haidar ${ }^{1}$, Mohammad N. Abdulrahim ${ }^{2} \S$ \\ ${ }^{1,2}$ Department of Mathematics \\ Beirut Arab University \\ P.O. Box 11-5020, Beirut, LEBANON
}

\begin{abstract}
Consider a representation $\rho: B_{3} \rightarrow G L_{6}(\mathbb{C})$ constructed by M. Al-Tahan and M. Abdulrahim. We construct a representation $\phi$ equivalent to the restriction of $\rho$ on $P_{3}$ and show that $\phi$ is a direct sum of irreducible subrepresentations, which are not equivalent to the reduced Burau representation restricted to $P_{3}$. Also, we show that the subrepresentations of $\phi$ are unitary relative to unique invertible hermitian matrices.
\end{abstract}

AMS Subject Classification: 20F36

Key Words: braid group, pure braid group, irreducible, unitary

\section{Introduction}

Let $B_{n}$ be the braid group on $n$ strands. There exists an obvious surjective group homomorphism $\pi: B_{n} \rightarrow S_{n}$. The kernel of $\pi$ is referred to as the pure braid group $P_{n}$ with $\frac{n(n-1)}{2}$ generators. Burau constructed a representations of $B_{n}$ of degrees $n$ and $n-1$, known as Burau and reduced Burau representations respectively [4]. The reduced Burau representation of $B_{n}$ was proved to be irreducible [5].

Also, researchers gave a great value for representations of the pure braid group $P_{n}$. M. Abdulrahim gave a necessary and sufficient condition for the irreducibility of the complex specialization of the reduced Gassner representation of

Received: December 12, 2018

(c) 2019 Academic Publications

$\S_{\text {Correspondence author }}$ 
$P_{n}$, [1]. Moreover, M. Al-Tahan and M. Abdulrahim constructed an irreducible representation of $B_{3}$ of degree 6 , namely, $\rho: B_{3} \rightarrow G L_{6}(\mathbb{C})$, [2]. In our work, we define a representation $\phi$ equivalent to the restriction of the representation $\rho$ of $B_{3}$ on $P_{3}$ and show that $\phi$ is a direct sum of three irreducible subrepresentations of degree two each, namely $\phi_{1}, \phi_{2}$, and $\phi_{3}$ (Theorem 2). Moreover, we show that each of $\phi_{1}, \phi_{2}$, and $\phi_{3}$ is not equivalent to the complex specialization of the reduced Burau representation restricted to $P_{3}$ of dimension 2 (Theorem 3 ). Finally we show that each of the irreducible subrepresentations of $\phi$ is unitary relative to a unique invertible hermitian matrix (Theorem 4).

\section{Definitions and preliminaries}

Definition 1. Let $\mathbb{C}^{r}=r \times 1$ (or column) vectors, $\overline{\mathbb{C}}^{r}=1 \times r$ (or row) vectors. A matrix $X \in M_{r}(\mathbb{C})$ is a pseudoreflection if $X-I$ has rank 1 . If $X$ is a pseudoreflection, then $X=I-A B$, where $A \in \mathbb{C}^{r}$ and $B \in \overline{\mathbb{C}}^{r}$.

Notation 1. Let $(*): M_{n}\left(\mathbb{C}\left[t^{ \pm 1}\right]\right)$ be an involution defined as follows:

$$
\left(h_{i j}(t)\right)^{*}=h_{j i}\left(t^{-1}\right), h_{i j}(t) \in \mathbb{C}\left[t^{ \pm 1}\right]
$$

Definition 2. Let $N$ and $U$ be elements of $G L_{n}(\mathbb{C}), U$ is called unitary relative to $N$ if $U N U^{\star}=N$.

Definition 3. ([3]) The braid group on $n$ strings, $B_{n}$, is the abstract group with presentation $B_{n}=\left\{\sigma_{1}, \ldots, \sigma_{n-1} ; \sigma_{i} \sigma_{i+1} \sigma_{i}=\sigma_{i+1} \sigma_{i} \sigma_{i+1}\right.$, for $i=$ $1,2, \ldots, n-2, \sigma_{i} \sigma_{j}=\sigma_{j} \sigma_{i}$ if $\left.|i-j|<1\right\}$.

The generators $\sigma_{1}, \ldots, \sigma_{n-1}$ are called the standard generators of $B_{n}$.

Definition 4. ([3]) The pure braid group, $P_{n}$, is defined as the kernel of the homomorphism $B_{n} \longrightarrow S_{n}$, defined by $\sigma_{i} \longrightarrow(i, i+1), 1 \leq i \leq n-1$. It has the following generators: $A_{i j}=\sigma_{j-1} \sigma_{j-2} \ldots \sigma_{i+1} \sigma_{i}^{2} \sigma_{i+1}^{-1} \ldots \sigma_{j-2}^{-1} \sigma_{j-1}^{-1}, 1 \leq i, j \leq n$. 


\section{Representations of $B_{3}$ and $P_{3}$}

\subsection{Reduced Burau representation of $B_{3}$ of dimension 2}

Definition 5. ([6]) The reduced Burau representation of the braid group on three strands $\beta_{3}: B_{3} \longrightarrow G L_{2}\left(Z\left[t^{ \pm 1}\right]\right)$, where $Z\left[t^{ \pm 1}\right]$ is a Laurent polynomial ring, is the matrix representation defined on the generators $\sigma_{1}, \sigma_{2}$ of $B_{3}$ by

$$
\beta_{3}\left(\sigma_{1}\right)=\left(\begin{array}{ll}
-t & 0 \\
-1 & 1
\end{array}\right), \beta_{3}\left(\sigma_{2}\right)=\left(\begin{array}{ll}
1 & -t \\
0 & -t
\end{array}\right)
$$

Proposition 1. ([5]) For $z \in \mathbb{C}^{*}$, the complex specialization of the reduced Burau representation of $B_{n}$, namely $\beta_{n}(z): B_{n} \longrightarrow G L_{n-1}(\mathbb{C})$, is irreducible if and only if $z$ is not a root of $f_{n}(t)=t^{n-1}+t^{n-2}+\ldots+t+1$.

Definition 6. For any $z \in \mathbb{C}^{*}, \beta_{3}(z): B_{3} \longrightarrow G L_{2}(\mathbb{C})$ is the representation obtained from $\beta_{3}$ by the specialization $t \rightarrow z$.

Definition 7. Let $\beta_{3}(z): P_{3} \longrightarrow G L_{2}(\mathbb{C})$ be the complex specialization of the reduced Burau representation restricted to the pure braid group $P_{3}$ defined as follows:

$$
\beta_{3}(z)\left(A_{12}\right) \rightarrow\left(\begin{array}{cc}
z^{2} & 0 \\
z-1 & 1
\end{array}\right), \beta_{3}(z)\left(A_{23}\right) \rightarrow\left(\begin{array}{cc}
1 & z(z-1) \\
0 & z^{2}
\end{array}\right)
$$

and

$$
\beta_{3}(z)\left(A_{13}\right) \rightarrow\left(\begin{array}{cc}
z & 1-z \\
-z(z-1) & z^{2}-z+1
\end{array}\right)
$$

Theorem 1. ([5]) Let $X_{1}=I-A_{1} B_{1}, \ldots, X_{r}=I-A_{r} B_{r}$ be $r$ invertible pseudoreflections in $M_{r}(\mathbb{C})$, where $r \geq 2$. Let $\Gamma$ be the directed graph whose vertices are $1,2, \ldots, r$, and which has a directed edge from $i$ to $j(i \neq j)$ precisely when $B_{i} A_{j} \neq 0$. Let $G$ be the subgroup of $G L_{r}(\mathbb{C})$ generated by $X_{1}, \ldots, X_{r}$. Then the following are equivalent:

1) $G$ is an irreducible subgroup of $G L_{r}(\mathbb{C})$.

2) For each $i \neq j$, with $1 \leq i, j \leq r$, the graph $\Gamma$ contains a directed path from $i$ to $j$, and $\left(B_{i} A_{j}\right) \in M_{r}(\mathbb{C})$ is invertible.

Proposition 2. ([7]) (Shur's Lemma) Suppose that $F$ is $n \times n$ matrix such that $F \alpha(g)=\alpha(g) F$ for every $g \in G$, where $\alpha$ is an irreducible representation 
of the group $G$. Then $F=\lambda I$ for some $\lambda \in \mathbb{C}$, where $I$ is the $n \times n$ identity matrix.

Proposition 3. The complex specialization of the reduced Burau representation restricted to $P_{3}$, namely $\beta_{3}(z)$, is irreducible if and only if $z^{3} \neq 1$.

Proof. Consider $U_{3}$ the free normal subgroup of $P_{3}$ with generatores $A_{13}$ and $A_{23}$. It is easy to see that $\beta_{3}(z)\left(A_{13}\right)$ and $\beta_{3}(z)\left(A_{23}\right)$ are pseudoreflections.

We can see that $\beta_{3}(z)\left(A_{13}\right)=I_{2}-A_{1} B_{1}$ and $\beta_{3}(z)\left(A_{23}\right)=I_{2}-A_{2} B_{2}$, where

$$
\begin{gathered}
A_{1}=\left(\begin{array}{c}
1 \\
-z
\end{array}\right), A_{2}=\left(\begin{array}{c}
z \\
z+1
\end{array}\right), B_{1}=\left(\begin{array}{cc}
1-z & z-1
\end{array}\right), \\
B_{2}=\left(\begin{array}{ll}
0 & 1-z
\end{array}\right) .
\end{gathered}
$$

Let $F$ be the inner product $\left\langle B_{i} A_{j}\right\rangle$. The determinant of $F$ equals to $z^{3}-1$. Thus $\beta_{3}(z)$ restricted to $U_{3}$ is irreducible if and only if $z^{3}-1 \neq 0$ (see Theorem 1). It follows that $\beta_{3}(z)$ restricted to $P_{3}$ is irreducible if $z^{3}-1 \neq 0$. Now, let us show if $z^{2}+z+1=0$ or $z-1=0$ then $\beta_{3}(z)$ restricted to $P_{3}$ is reducible. If $z-1=0$ then $A_{12}=A_{13}=A_{23}=I_{2}$. Thus it is reducible.

Otherwise, if $z^{2}+z+1=0$ then the reducibility on $P_{3}$ follows from reducibility on $B_{3}$ (see Proposition 1).

\subsection{Representation of $B_{3}$ of Dimension 6}

A new six dimensional representation of $B_{3}$ was constructed by M. Al-Tahan and M. Abdulrahim.

Definition 8. ([2]) Let $z$ be a non zero complex number with $z^{2} \neq 1$. We consider the representation $\rho$ of $B_{3}$ given by

$$
\rho\left(\sigma_{1}\right)=\left(\begin{array}{cccccc}
1-z & z & 0 & 0 & 0 & 0 \\
1 & 0 & 0 & 0 & 0 & 0 \\
0 & 0 & 0 & z^{-1} & 0 & 0 \\
0 & 0 & z & 0 & 0 & 0 \\
0 & 0 & 0 & 0 & 0 & 1 \\
0 & 0 & 0 & 1-z^{-1} & 1 & 0
\end{array}\right)
$$


and

$$
\rho\left(\sigma_{2}\right)=\left(\begin{array}{cccccc}
0 & 0 & 1 & 0 & 0 & 0 \\
0 & 0 & z^{-1} & 0 & 0 & z^{-1} \\
1 & z-1 & 0 & 0 & 0 & 0 \\
0 & 0 & 0 & 1 & z & 0 \\
0 & 0 & 0 & 0 & -z & 0 \\
-1 & 1 & 0 & 0 & 1 & 0
\end{array}\right)
$$

Proposition 4. ([2]) The representation of $B_{3}, \rho: B_{3} \rightarrow G L_{6}(\mathbb{C})$ is irreducible.

\section{Reducibility of the representation $\rho$ restricted to $P_{3}$}

We construct a representation $\phi$ equivalent to the restriction of $\rho$ on $P_{3}$. More precisely, we conjugate by the matrix

$$
T=\left(\begin{array}{llllll}
1 & 0 & 0 & 0 & 0 & 0 \\
0 & 1 & 0 & 0 & 0 & 0 \\
0 & 0 & 1 & 0 & 0 & 0 \\
0 & 0 & 0 & 0 & 1 & 0 \\
0 & 0 & 0 & 0 & 0 & 1 \\
0 & 0 & 0 & 1 & 0 & 0
\end{array}\right)
$$

It is easy to see that $\phi$ is a direct sum of three representations, namely $\phi_{1}, \phi_{2}$, and $\phi_{3}$. Then we prove that $\phi_{1}, \phi_{2}$, and $\phi_{3}$ are irreducible. Moreover, we show that each of $\phi_{1}, \phi_{2}$, and $\phi_{3}$ is not equivalent to the complex specialization of the reduced Burau representation restricted to $P_{3}$ of dimension 2. Finaly we show that $\phi_{1}, \phi_{2}$, and $\phi_{3}$ are unitary relative to unique invertible hermitian matrices.

Definition 9. let $z$ be a non-zero complex number, with $z^{2} \neq 1$. Consider $\phi: P_{3} \rightarrow G L_{6}(\mathbb{C})$, defined as follows:

$$
\phi\left(A_{12}\right)=\left(\begin{array}{cccccc}
z^{2}-z+1 & -z(z-1) & 0 & 0 & 0 & 0 \\
-z+1 & z & 0 & 0 & 0 & 0 \\
0 & 0 & 1 & 0 & 0 & 0 \\
0 & 0 & z-1 & 1 & 0 & 0 \\
0 & 0 & 0 & 0 & 1 & 0 \\
0 & 0 & 0 & 0 & \frac{z-1}{z} & 1
\end{array}\right),
$$




$$
\phi\left(A_{23}\right)=\left(\begin{array}{cccccc}
1 & z-1 & 0 & 0 & 0 & 0 \\
0 & 1 & 0 & 0 & 0 & 0 \\
0 & 0 & \frac{2 z-1}{z} & \frac{z-1}{z} & 0 & 0 \\
0 & 0 & \frac{1-z}{z} & z^{-1} & 0 & 0 \\
0 & 0 & 0 & 0 & 1 & -z(z-1) \\
0 & 0 & 0 & 0 & 0 & z^{2}
\end{array}\right)
$$

and

$$
\phi\left(A_{13}\right)=\left(\begin{array}{cccccc}
1 & 0 & 0 & 0 & 0 & 0 \\
\frac{z-1}{z} & 1 & 0 & 0 & 0 & 0 \\
0 & 0 & 1 & 1-z & 0 & 0 \\
0 & 0 & 0 & z^{2} & 0 & 0 \\
0 & 0 & 0 & 0 & z & z-1 \\
0 & 0 & 0 & 0 & 1-z & 2-z
\end{array}\right)
$$

Theorem 2. The representation $\phi$ is a direct sum of irreducible subrepresentations.

Proof. The representation $\phi$ is reducible because there is an invariant subspace spanned by $e_{1}$ and $e_{2}$. Moreover, we write $\phi=\phi_{1} \oplus \phi_{2} \oplus \phi_{3}$, where $\phi_{1}$, $\phi_{2}$, and $\phi_{3}$ are given by

$$
\begin{gathered}
\phi_{1}\left(A_{13}\right)=\left(\begin{array}{cc}
1 & 0 \\
\frac{z-1}{z} & 1
\end{array}\right), \phi_{1}\left(A_{12}\right)=\left(\begin{array}{cc}
z^{2}-z+1 & -z(z-1) \\
-z+1 & z
\end{array}\right), \\
\phi_{1}\left(A_{23}\right)=\left(\begin{array}{cc}
1 & z-1 \\
0 & 1
\end{array}\right), \\
\phi_{2}\left(A_{12}\right)=\left(\begin{array}{cc}
1 & 0 \\
z-1 & 1
\end{array}\right), \phi_{2}\left(A_{23}\right)=\left(\begin{array}{cc}
\frac{2 z-1}{z-z} & \frac{z-1}{z} \\
\frac{1-1}{z} & z^{-1}
\end{array}\right), \\
\phi_{2}\left(A_{13}\right)=\left(\begin{array}{cc}
1 & 1-z \\
0 & z^{2}
\end{array}\right) \\
\phi_{3}\left(A_{12}\right)=\left(\begin{array}{cc}
1 & 0 \\
\frac{z-1}{z} & 1
\end{array}\right), \phi_{3}\left(A_{23}\right)=\left(\begin{array}{cc}
1 & -z(z-1) \\
0 & z^{2}
\end{array}\right), \\
\phi_{3}\left(A_{13}\right)=\left(\begin{array}{cc}
z & z-1 \\
1-z & 2-z
\end{array}\right) .
\end{gathered}
$$


Now, we use Theorem 1 to show that $\phi_{1}, \phi_{2}$, and $\phi_{3}$ are irreducible. Consider $U_{3}$ the free normal subgroup of $P_{3}$ with generatores $A_{13}$ and $A_{23}$. It is easy to see that $\phi_{k}\left(A_{13}\right)$ and $\phi_{k}\left(A_{23}\right)$ are pseudoreflections, where $k \in\{1,2,3\}$. We see that

$$
\phi_{k}\left(A_{13}\right)=I_{2}-A_{1}^{(k)} B_{1}^{(k)}, \phi_{k}\left(A_{23}\right)=I_{2}-A_{2}^{(k)} B_{2}^{(k)},
$$

where

$$
\begin{gathered}
A_{1}^{(1)}=\left(\begin{array}{c}
0 \\
\frac{1-z}{z}
\end{array}\right), A_{2}^{(1)}=\left(\begin{array}{c}
1-z \\
0
\end{array}\right), B_{1}^{(1)}=\left(\begin{array}{ll}
1 & 0
\end{array}\right), \\
B_{2}^{(1)}=\left(\begin{array}{ll}
0 & 1
\end{array}\right) . \\
A_{1}^{(2)}=\left(\begin{array}{c}
-1 \\
1+z
\end{array}\right), A_{2}^{(2)}=\left(\begin{array}{c}
1-z \\
z-1
\end{array}\right), B_{1}^{(2)}=\left(\begin{array}{ll}
0 & 1-z
\end{array}\right), \\
B_{2}^{(2)}=\left(\begin{array}{ll}
1 / z & 1 / z
\end{array}\right) . \\
A_{1}^{(3)}=\left(\begin{array}{c}
1-z \\
z-1
\end{array}\right), A_{2}^{(3)}=\left(\begin{array}{c}
-z \\
1+z
\end{array}\right), B_{1}^{(3)}=\left(\begin{array}{ll}
1 & 1
\end{array}\right), \\
B_{2}^{(3)}=\left(\begin{array}{ll}
0 & 1-z
\end{array}\right) .
\end{gathered}
$$

Let $F_{k}$ be the inner product $\left\langle B_{i}^{(k)} A_{j}^{(k)}>\right.$. It is easy to see that $\operatorname{det}\left(F_{1}\right)=$ $\frac{-(z-1)^{2}}{z} \neq 0$ and $\operatorname{det}\left(F_{2}\right)=\operatorname{det}\left(F_{3}\right)=(z-1)^{2} \neq 0$. Thus $\phi_{k}$ 's, restricted to $U_{3}$, are irreducible (Theorem 1). It follows that $\phi_{1}, \phi_{2}$, and $\phi_{3}$ are irreducible.

Theorem 3. The representations $\phi_{1}, \phi_{2}$, and $\phi_{3}$ are not equivalent to the complex specializations of the irreducible reduced Burau representation restricted to the pure braid group $P_{3}$.

Proof. For any non-zero complex number $z$ with $z^{2} \neq 1$, it is easy to see that the images of the generators of $P_{3}$ under the complex specialization of the irreducible reduced Burau representation restricted to $P_{3}$ have two distinct eigenvalues 1 and $z^{2}$ (see Definition 7). But each of the images of $A_{23}$ under $\phi_{1}$, and $A_{12}$ under $\phi_{2}$ and $\phi_{3}$ has only one eigenvalue equals to 1 .

It was shown that the reduced Burau representation of the braid group is unitary relative to a hermitian matrix (see [8]). We show that $\phi_{1}, \phi_{2}$, and $\phi_{3}$, which are irreducible, are unitary relative to hermitian matrices. Thus, we get the following theorem. 
Theorem 4. The representations $\phi_{1}, \phi_{2}$, and $\phi_{3}$ are unitary relative to unique invertible hermitian matrices $N_{1}, N_{2}$, and $N_{3}$ respectively.

Proof. We define the matrices $N_{1}, N_{2}$, and $N_{3}$ as follows:

$$
\begin{gathered}
N_{1}=\left(\begin{array}{cc}
0 & z+1 \\
z^{-1}+1 & 0
\end{array}\right), N_{2}=\left(\begin{array}{cc}
0 & z^{-1}+1 \\
z+1 & \frac{-(z+1)^{2}}{z}
\end{array}\right), \\
N_{3}=\left(\begin{array}{cc}
0 & z+1 \\
z^{-1}+1 & \frac{-(z+1)^{2}}{z}
\end{array}\right) .
\end{gathered}
$$

For $i \in\{1,2,3\}, \operatorname{det}\left(N_{i}\right)=\frac{-(z+1)^{2}}{z} \neq 0$. Also, it is easy to see that $N_{i}^{*}=N_{i}$. Thus $N_{i}$ 's are invertible and hermitian. We also have for $i \in\{1,2,3\}$

$$
\begin{gathered}
\phi_{i}\left(A_{12}\right) N_{i}\left(\phi_{i}\left(A_{12}\right)\right)^{*}=N_{i}, \phi_{i}\left(A_{23}\right) N_{i}\left(\phi_{i}\left(A_{23}\right)\right)^{*}=N_{i}, \\
\phi_{i}\left(A_{13}\right) N_{i}\left(\phi_{i}\left(A_{13}\right)\right)^{*}=N_{i} .
\end{gathered}
$$

Moreover, $\phi_{i}$ 's are irreducible (Theorem 2), and the uniqueness of $N_{i}$ 's, up to scalar multiplication, follows from Shur's lemma.

\section{References}

[1] M.N. Abdulrahim, Complex specializations of the reduced Gassner representation of the pure braid group, Proc. of American Mathematical Society, 125, No 6 (1997), 1617-1624.

[2] M. Al-Tahan, M.N. Abdulrahim, A new six dimensional representation of the braid group on three strands and its irreducibility and unitarizability, British J. of Mathematics and Computer Science, 3, No 3 (2013), 275-280.

[3] J.S. Birman, Braids, Links and Mapping Class Groups, Annals of Mathematical Studies \# 82, Princeton Univ. Press, New Jersy (1975).

[4] W. Burau, Uber Zopfgruppen und gleischsinning verdrillte Verkettungen, Abh. Math. Sem. Ham. II (1936), 171-178.

[5] E. Formanek, Braid group representations of low degree, Proc. London Math. Soc., 73, No 3 (1996), 279-322.

[6] V.F.R. Jones, Hecke algebra representations of braid groups and link polynomials, Ann. of Math., 126 (1987), 335-388. 
[7] I. Schur, Neue Begründung der Theorie der Gruppencharaktere, Sitzungsberichte der Königlich Preußischen Akademie der Wissenschaften zu Berlin (1905), 406-432.

[8] C. Squier, The Burau representation is unitary, Proc. Amer. Math. Soc., 90 (1984), 199-202. 
